\title{
Curvature Effects in GRBs
}

\section{A. Shenoy ${ }^{* 1}$ E. Sonbas, ${ }^{2}{ }^{6}$ C. D. Dermer, ${ }^{3}$ L. C. Maximon, ${ }^{1}$ K. S. Dhuga, ${ }^{1}$ J. Hakkila, ${ }^{4}$ P. N. Bhat,${ }^{5}$ W. C. Parke,${ }^{1}$ G. Maclachlan, ${ }^{1}$ T. N. Ukwatta. ${ }^{7}$}

${ }^{1}$ Department of Physics, The George Washington University Washington, D.C. 20052, USA

${ }^{2}$ Department of Physics, University of Adiyaman 02040, Adiyaman, Turkey

${ }^{3}$ Space Science Division, Code 7653, Naval Research Laboratory Washington, D.C. 20375, USA

${ }^{4}$ Department of Physics and Astronomy, College of Charleston Charleston, S.C. 29424, USA

${ }^{5}$ Physics Department, University of Alabama in Huntsville Huntsville, AL 35805, USA

${ }^{6}$ NASA Goddard Space Flight Center

Greenbelt, MD 20771, USA

${ }^{7}$ Department of Physics and Astronomy, Michigan State University East Lansing, MI 48824, USA

E-mail: ashwinsp469@gmail.com, edasonbas@yahoo.com, charles.dermer@nrl.navy.mil, maxegwu.edu, dhuga@gwu.edu, hakkilajecofc.edu, Narayana.Bhat@nasa.gov, wparke@gwu.edu, maclach@gwu.edu, tilan.ukwatta@gmail.com

\begin{abstract}
Spectral lags in Gamma-ray Bursts (GRBs), wherein low-energy photons arrive at later times than the high-energy photons, have been observed in a significant number of bursts. Spectral lags have been attributed to curvature effects in GRBs. In this scenario, the relativistic GRB jet is assumed to be a cone with a certain opening angle. Photons that are off-axis relative to the observer arrive at later times than on-axis photons. In this work, we test GRB data for spectral lags and other such curvature effects by invoking a relatively simple kinematic, two-shell collision model for a uniform jet profile. We have isolated single pulse structures in the Fermi-GBM, BATSE, and Swift-BAT GRB samples. We present the preliminary results of our analysis.
\end{abstract}

Gamma-Ray Bursts 2012 Conference -GRB2012,

May 07-11, 2012

Munich, Germany

\footnotetext{
${ }^{*}$ Speaker.
} 


\section{Introduction}

Pulses in GRB light curves are thought to be produced by collisions between relativistic shells ejected from an unsteady wind from the central engine [1]. Such pulses have particular observable signatures that can be understood when effects from relativistic time delays in the radiating shells and the emission properties of the jet are taken into account. Among such observational signatures are the so called "curvature effects", which are primarily kinematical effects, and are thought to occur due to the emission of photons from high latitudes in the emission region of the colliding shells. One such testable prediction is the peak-flux - peak-frequency relation which dictates that the peak photon energy $\varepsilon_{p k}$, where the $v F_{v}$ flux reaches its maximum value, is proportional to $\varepsilon_{p k}^{3}$ in the declining phase of a GRB pulse [3]. Another well-known phenomenon sometimes attributed to curvature effects and seen in a significant fraction of long GRBs, is the spectral lag wherein, low-energy photons arrive at later times than the high-energy photons.

\section{The Model}

In previous research [3], the kinematic properties of a uniform spherical shell traveling with bulk Lorentz factor $\Gamma$ that radiates for a comoving time between $t_{0}^{\prime}$ and $t_{0}^{\prime}+\Delta t^{\prime}$ was studied. The comoving width of the shell $\Delta r^{\prime}$ is assumed to remain constant during the period of illumination, and the emitted spectrum is described by a broken power-law peaking at a comoving photon energy $\varepsilon_{p k, i}^{\prime}$.

If curvature effects dominate pulse formation, three generic types of GRB pulses are formed, depending on the relative values of $c \Delta t^{\prime}, \Delta r^{\prime}$, and the radius $r_{0}$ when the illumination begins.

In Fig. 1 we show the evolution of the spectral energy distribution for the standard case (for additional details see [3]). Notice the rapid decay $\propto \varepsilon_{p k}^{3}$ of the $v F_{v}$ peak flux $f_{\varepsilon_{p k}}$ measured at $\varepsilon_{p k}$ during the decay portion of the pulse, which holds in the declining phase for all three types of pulses. Another prediction of the model is that a spectral lag should be present for energies below $\varepsilon_{p k, i}$ (The $\varepsilon_{p k}$ of the spectrum at the beginning of the pulse), while no lag is expected for energies above $\varepsilon_{p k, i}$ for the assumed intrinsic broken-power-law spectrum.

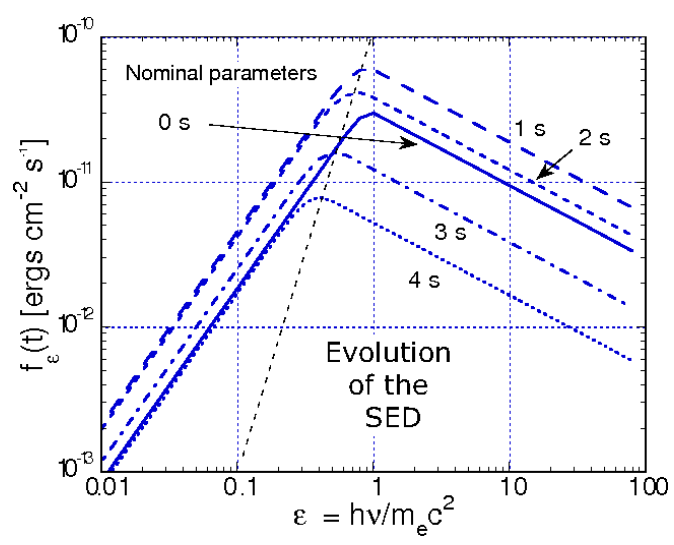

Figure 1: Evolution of the spectral energy distribution due to curvature effects. In the declining phase of the pulse, the value of $f_{\varepsilon_{p k}} \propto \varepsilon_{p k}^{3}$, as shown by the dashed line.

\section{Methodology}

Given the relative paucity of bright, clean single-pulse GRBs in the GBM sample, one potentially beneficial strategy is to isolate relatively distinct pulse structures within bright GRBs that contain multiple pulses ( $\sim 25 \%$ of all long bursts). We thus propose the following methodology. 
Testing the $f_{\varepsilon_{p k}} \propto \varepsilon_{p k}^{3}$ Relation:

1. Identify and fit bright structures in GRBs using one of the standard pulse functions (Norris pulse function, log-normal, etc.). Identify pulses that can be isolated temporally and spectrally.

2. Divide the pulses into appropriate time segments.

3. Fit the spectrum of each time segment with the best-fit spectral function and extract $\varepsilon_{p k}$ and it's error.

4. Extract the $v F_{v}$ flux $\left(f_{\varepsilon_{p k}}\right)$ and flux error in the range centered at $\varepsilon_{p k}$ and spanned by its error.

5. Test if $f_{\varepsilon_{p k}}$ is proportional to $\varepsilon_{p k}^{3}$ in the decay portion of the pulse.

\section{Testing the Model against Spectral Lags:}

1. Identify $\varepsilon_{p k, i}$ for isolated pulses using spectral fits.

2. Extract light curves in suitable energy bands.

3. Extract lags between these light curves using the Cross Correlation Function (CCF) method [4].

4. Modify key parameters in the model such as $\varepsilon_{p k, i}$ and $\mathrm{z}$ (if known) to match observations.

5. Modify the shell parameters such as $c \Delta t^{\prime}, r^{\prime}$, and $r_{0}$ to generate a comparable pulse profile.

6. Extract theoretical lags between light curves in identical energy bands and compare predictions with observations.

\section{Results}
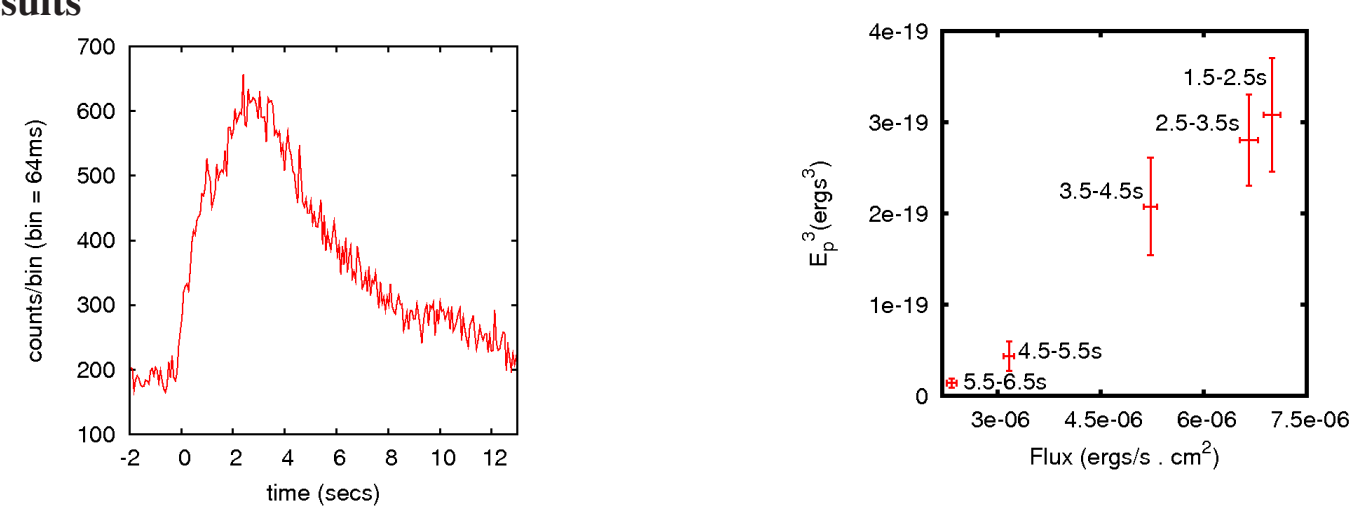

Figure 2: Light curve for the GBM GRB 081224887.

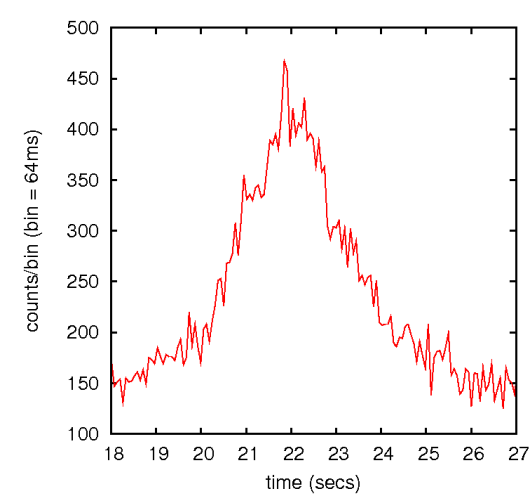

Figure 4: Light curve for the GBM GRB 090718762.

Figure 3: $f_{\varepsilon_{p k}}$ vs. $\varepsilon_{p k}^{3}$ for GRB 081224887

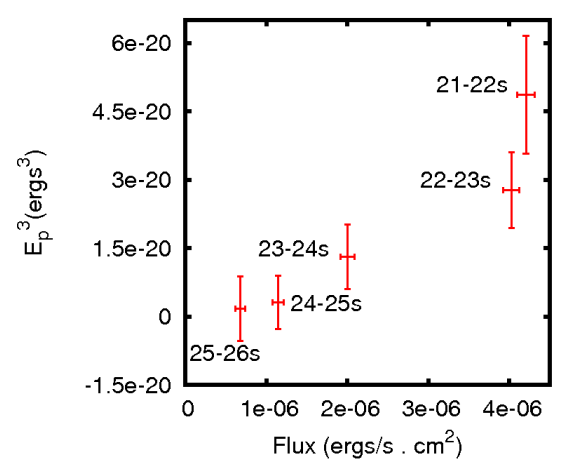

Figure 5: $f_{\varepsilon_{p k}}$ vs. $\varepsilon_{p k}^{3}$ for GRB 090718762.

In figures $2-5$, we present the results of our analysis for two Fermi-GBM GRBs. The light curves were extracted over the entire GBM range $(8 \mathrm{keV}-\sim 40 \mathrm{MeV})$. The spectra for both GRBs were found to be best fit by the Band function. It can be seen in Figs. 3 and 5, that the relation 
holds over a certain range of energies in the decay portion of the pulses. It should also be pointed out that curvature effects can account for the steep decay phase seen in the x-ray observations of some GRBs by Swift. There are some GRBs that display possible evidence of a photospheric emission component, and this indeed may account for the short variabilities (of order ms) seen in a significant fraction of GRBs, and which may be independent of variabilities produced by colliding shells. Detailed spectral analysis to search for a photospheric component might be able to distinguish emission from colliding shells at large size scales and quasi-thermal emissions at small size scales. We note that, in order to produce the pulses seen in the light curves presented in the paper using two-shell collisions, the radii need to be of order $10^{15}-10^{16} \mathrm{~cm}$. These results are in agreement with Hascoet et al. [2], who have compared the internal shock model to the photospheric and the magnetic reconnection models and have concluded that the high latitude emissions from the last colliding shells in the prompt emission, i.e. the internal shock model, is the leading model to explain this steep decay phase.

We tested the prediction against the spectral lags extracted for GRB 081224887 using the CCF method. The lags were extracted in a temporal range of $2.5-5.5$ seconds and at energies $\leq 200 \mathrm{keV}$.

In Fig. 6 we show the spectral lags extracted for both the theoretical model and the observations for GRB 081224887 (using the CCF method for both sets of light curves). Identical energy bands were chosen for both sets of light curves. The model parameters were modified to obtain a good fit between theory and observations. We see that the lag evolution matches the predicted trend closely.

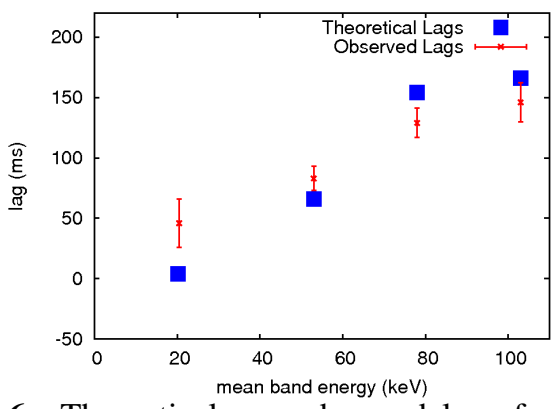

Figure 6: Theoretical vs. observed lags for GRB 081224887. The energy plotted is the arithmetic mean of the centers of the energies of the individual bands.

\section{Summary and Conclusions}

We have searched for curvature effects in the prompt emission of GRBs by testing the predictions of a simple kinematical 2-shell model for the temporal evolution of GRB spectra via the peak-flux - peak-frequency relation $\left(f_{\varepsilon_{p k}} \propto \varepsilon_{p k}^{3}\right)$, and the spectral lags. We chose a small sample of Fermi-GBM GRBs for this purpose and found that the $f_{\varepsilon_{p k}} \propto \varepsilon_{p k}^{3}$ relations holds well for a certain region in the decay portion of the pulses. For one GRB in this sample, we were able to obtain an excellent match between theory and data for the spectral lags. We thus find that there is sufficient evidence for curvature effects in GRBs and intend to continue this analysis for the Fermi-GBM GRBs while also extending our analysis to the Swift and BATSE GRB samples.

\section{References}

[1] Rees, M. J., \& Meszaros, P. 1994, ApJ, 430, L93.

[2] Hascoet, R., Daigne, F., Mochkovitch, R. 2012, A\&A, 549, L29.

[3] Dermer, C. D. 2004, ApJ, 614, 284.

[4] Ukwatta, T. N. et al., 2010a, MNRAS, 711, 1073. 